\title{
Evaluation of redundancy analysis to identify signatures of local adaptation
}

Thibaut CAPBLANCQ1 ${ }^{*}$, Keurcien LUU2 ${ }^{2}$, Michael G.B. BLUM² and Eric BAZIN1,\#

${ }^{1}$ Univ. Grenoble Alpes, CNRS, LECA UMR 5553, Grenoble, France

${ }^{2}$ Univ. Grenoble Alpes, CNRS, TIMC-IMAG UMR 5525, Grenoble, France

\#Deceased

* Corresponding author: Thibaut Capblancq, 2233 Rue de la Piscine, 38041 Grenoble Cedex, France. Tel.: +33 (0)4 765136 71, e-mail: thibaut.capblancq@univ-grenoble$\underline{\text { alpes.fr }}$

Date: May 18, 2018

Running title: RDA-based procedure for genome scans 


\section{ABSTRACT}

Ordination is a common tool in ecology that aims at representing complex

3 biological information in a reduced space. In landscape genetics, ordination methods

4 such as principal component analysis (PCA) have been used to detect adaptive

5 variation based on genomic data. Taking advantage of environmental data in

6 addition to genotype data, redundancy analysis (RDA) is another ordination

7 approach that is useful to detect adaptive variation. This paper aims at proposing a

8 test statistic based on RDA to search for loci under selection. We compare

9 redundancy analysis to pcadapt, which is a nonconstrained ordination method, and

10 to a latent factor mixed model (LFMM), which is a univariate genotype-environment

11 association method. Individual-based simulations identify evolutionary scenarios

12 where RDA genome scans have a greater statistical power than genome scans based

13 on PCA. By constraining the analysis with environmental variables, RDA performs

14 better than PCA in identifying adaptive variation when selection gradients are

15 weakly correlated with population structure. Additionally, we show that if RDA and

16 LFMM have a similar power to identify genetic markers associated with

17 environmental variables, the RDA-based procedure has the advantage to identify the

18 main selective gradients as a combination of environmental variables. To give a

19 concrete illustration of RDA in population genomics, we apply this method to the

20 detection of outliers and selective gradients on an SNP data set of Populus 
21 trichocarpa (Geraldes et al., 2013). The RDA-based approach identifies the main

22 selective gradient contrasting southern and coastal populations to northern and

23 continental populations in the northwestern American coast.

25 KEYWORDS: Genome scans - Multivariate analysis - Redundancy analysis -

26 Biological adaptation - Selection - Environmental variables

28 INTRODUCTION

Natural selection results from environmental pressures. The environment acts

30 simultaneously on several characteristics, and these adaptive characteristics can be

31 determined by a large number of alleles of small effects (Pritchard and Di Rienzo,

32 2010). Patterns of adaptive variation within species are usually studied through

33 physiological, morphometric and fitness comparisons in common garden

34 experiments. However, this is a difficult and constraining task, which can even be

35 unrealistic to achieve in species with a long generation time. Recently, the

36 development of next-generation sequencing (NGS) technologies has opened the

37 possibility to access to a large amount of genetic variation across the genome.

38 Therefore, relationships between genetic polymorphism, phenotypic variation and

39 environmental variables can now be studied and quantified by in situ approaches

40 (Steane et al., 2014, Vangestel et al., 2018). 
By looking for loci that are either excessively differentiated between

43 populations or significantly associated with environmental gradients, adaptive

44 variation can be detected (Foll and Gaggiotti 2008, Frichot et al., 2013, Vatsiou et al.,

45 2015, Duforet-Frebourg et al., 2016, Hoban et al., 2016).

46 In many genome scan procedures, outlier loci are identified based on genetic

47 differentiation, assuming that adaptive alleles have increased genetic differentiation.

48 A common statistic to evaluate genetic differentiation is $F_{\text {st }}$ (Lewontin and Krakauer,

49 1973) and many likelihood or Bayesian methods use $F_{s t}$-related statistics to scan

50 genomes for local adaptation (Bazin et al., 2010, De Villemereuil and Gaggiotti, 2015,

51 Foll and Gaggiotti, 2008, Whitlock and Lotterhos, 2015). One limitation of $\mathrm{F}_{\mathrm{st}}$-based

52 approaches is that they require defining discrete populations, whereas many species

53 are not structured in different populations but display continuous genetic gradients

54 (Martins et al. 2016). In these cases, defining populations can be challenging and can

55 directly interfere with the result of the analysis (Yang et al., 2012).

A solution to avoid such an issue is to use individual-based multivariate

57 methods such as PCA that ascertain population structure. Duforet-Frebourg and

58 colleagues (2016) proposed a genome scan method based on PCA (pcadapt), where

59 outlier loci are the ones excessively correlated with one or more ordination axes

60 (Duforet-Frebourg et al., 2016). Simulations show that pcadapt has a similar power 
61 to model-based methods under island models and perform better when the

62 simulated demographic model drifts from the island model, for instance under

63 hierarchical population structure or isolation by distance patterns (Luu et al., 2017).

64 Nevertheless, one conundrum of such approaches is the difficulty to interpret

65 ordination axes in terms of ecological meanings. These are usually tight to

66 geographical axes (latitudinal or longitudinal), but they are not necessarily linked to

67 an environmental variable such as temperature, drought, diet habit, etc. When

68 environmental information exists, it has to be used, a posteriori, as a means of

69 interpretation, but it is not involved in the inference process.

70 Genotype-environment association (GEA) methods are based on a different

71 principle than methods based on genetic differentiation; they assume that adaptive

72 loci are significantly associated with environmental variation. These methods aim at

73 detecting alleles that are associated with environmental variables (e.g., temperature

74 and drought) with the idea that these alleles may confer a selective advantage in

75 some environment (Coop et al., 2010, Frichot et al., 2013). Most of these methods

76 only use one predictor variable at a time, which can be problematic when the main

77 selective gradient is unknown or when we want to disentangle multifactorial

78 associations between genetic and environmental variation. 
81 as RDA (redundancy analysis) or CAPs (canonical analysis of principal coordinates)

82 to combine the advantages of both multivariate methods and genotype-environment

83 association procedures (Lasky et al., 2012, DeKort et al., 2014; Steane et al., 2014,

84 Hecht et al., 2015). This family of methods subsequently aligns adaptive variation

85 with environmental data in order to be able to identify the environmental gradients

86 that are the most correlated with adaptive variation. In Arabidopsis thaliana, loci

87 involved in adaptation to climate have been found using RDA (Lasky et al., 2012).

88 Outliers were identified as SNPs with the greatest squared loadings along the first

89 RDA axes (i.e., those in the $0.5 \%$ tail). RDA can also be used to derive an adaptive

90 index that predicts the performance of individuals in different environmental

91 conditions. Steane et al. (2014) applied a similar approach (using canonical analysis

92 of principal coordinates) to Eucalyptus tricarpa populations and showed that both

93 detection of adaptive variation with RDA and common garden experiments provided

94 evidence for local adaptation. Finally, in two recent studies, Forester and colleagues

95 (2015 and 2017) tested the power of an RDA-based method for detecting signatures

96 of local adaptation in a heterogeneous landscape (Forester et al, 2015) and in

97 comparison with other constrained ordination methods (Forester et al, 2017). Using

98 simulations, they found that an RDA-based method has a superior combination of

99 low false positive and high true positive rates when compared to generalized linear

100 models (GLM) or latent factor mixed models (LFMM). 
102 statistical test for searching loci under selection. We use simulations and real data

103 sets to shed light on the conditions within which RDA is the most efficient and to

104 document the possibilities given by RDA when studying landscape genomics. We

105 also compare results of a genome scan based on RDA with genome scans obtained

106 with pcadapt and LFMM (Frichot et al. 2013, Luu et al., 2017). This investigation

107 gives the opportunity to show the advantages of a constrained ordination method

108 such as RDA in comparison with nonconstrained ordination method (PCA) and

109 univariate genotype-environment association method (LFMM). Finally, to illustrate

110 the pertinence of such a method in a concrete example, we apply it to a Populus

111 trichocarpa SNP dataset obtained from Geraldes et al. (2013). We found a large

112 overlap between the outlier loci detected with RDA and the loci previously detected

113 with Fst based methods (Geraldes et al., 2013). In addition, RDA revealed what the

114 main selective gradients are along the natural populations of Populus trichocarpa in

115 the northwestern coast of North America.

117 MATERIALS AND METHODS

118 1. Genome scan using an RDA-based approach

119 Redundancy analysis (RDA) was first introduced by Rao (1964). It is the

120 extension of multiple regressions to the modeling of multivariate response data 
121 (Legendre and Legendre 2012, section 11.1). The data are separated in two sets, a

122 response matrix $\mathrm{Y}$ of the variable to be explained (e.g., species abundance in a set of

123 sites; $m$ sites and $n$ species) and an explanatory matrix X (e.g., a set of environmental

124 variables within each site; $m$ sites and $p$ environment). In the following analysis, loci

125 replace species abundances and individuals replace sites. RDA seeks to project the

126 genetic variation between individuals that is explained by environmental data on a

127 reduced space. RDA assumes that there are linear relationships between the

128 response $(\mathrm{Y})$ and explanatory $(\mathrm{X})$ variables.

129 The analysis starts using a classical RDA procedure performed with the

130 function $r d a$ of the R package "vegan" (Oksanen et al., 2015). An individual genetic

131 dataset is used as the response matrix $Y$, and a set of environmental variables is used

132 as explanatory matrix X. We assume in the following that genotypes are coded using

133 the values 0,1 , or 2 that correspond to homozygote for the most frequent allele,

134 heterozygote and homozygote for the less frequent allele. RDA amounts at

135 constructing a matrix $Y^{\prime}$ of fitted genetic values estimated from the regression of

136 each locus by the environmental variables and at performing principal component

137 analysis on the matrix Y' (Legendre and Legendre 2012, section 11.1). After this

138 constrained ordination step, we follow the methodology implemented in pcadapt to

139 find outlier loci (Luu et al., 2017). First, we recover the loci loadings from the RDA

140 analysis. Only the loadings of the most informative ordination axis are kept for the 
141 rest of the procedure. The number of axes used $(\mathrm{K})$ is determined by looking at the

142 amount of information retained on the different axes of the RDA. A Mahalanobis

143 distance $\mathrm{D}$ is then computed for each locus to identify loci showing extreme D values

144 compared to the rest of the SNPs. A Mahalanobis distance is a multidimensional

145 generalization of the idea of measuring how many standard deviations is a point

146 from an average point. Computation of the Mahalanobis distance uses the covRob

147 function of the "robust" R package (Wang et al., 2014). Mahalanobis distances are

148 distributed as chi-squared distribution with $\mathrm{K}$ degrees of freedom after correcting

149 with the genomic inflation factor (Luu et al., 2017). Inflation factors are constant

150 values that are used to rescale chi-square statistics in order to limit inflation due to

151 diverse confounding factors (François et al 2016). We then adjust the resulting p-

152 values for the false discovery rate (FDR) by computing q-values with the "qvalue" $\mathrm{R}$

153 package (Dabney and Storey, 2011). A locus is considered as an outlier if its q-value

154 is less than $10 \%$, meaning that $10 \%$ or less of the identified outliers could be false

155 positives. The complete procedure is described in Fig. 1 and our R script is available

156 in the supplementary material (Script S1).

157 To evaluate the capacity of the RDA-based approach to detect loci underlying

158 environmental selective pressure, we first test the method with simulated datasets.

159 A total of 100 independent runs of simulations were used (see below for details). We

160 evaluate the power of the procedure based on RDA to detect positive loci (loci 
161 simulated under selection) and to return a controlled number of false positives

162 (neutral loci detected as outliers by the analysis). Then, to emphasize the utility of

163 this method, we compared it to the PCA-based procedure implemented in pcadapt

164 that does not use environmental variables (Luu et al., 2017). In the same vein, we

165 compare the multivariate RDA-based procedure to LFMM, which is an univariate

166 genotype-environment association method (Frichot et al., 2013). This method

167 processes one environmental variable at a time, whereas we wanted to evaluate to

168 what extent different techniques can disentangle covariation between multiple

169 environmental gradients and multilocus genetic variation. To tackle this issue, we

170 performed the LFMM analysis with a set of composite environmental variables

171 corresponding to the first two PCs of a PCA performed on the environmental

172 simulated variables (Frichot et al., 2013). It gave us orthogonal composite

173 environmental variables for which we tested covariation with genetic variability

174 through the LFMM procedure. The LFMM analysis was launched with only one latent

175 factor $(K=1)$, taking into account that simulations have been made without any

176 population divergence.

177

178 2. Simulated data

179 We used simulations performed with the simuPop python library (Peng et al.,

180 2005). A lattice of $8 \times 8$ populations is simulated (i.e., 64 populations in total). Each 
181 population is initialized with 200 diploid individuals with random genotypes.

182 Migration is set to 0.1 , resulting in an isolation-by-distance pattern across the

183 species range. Loci are assumed to be biallelic SNPs. The allele frequency of the

184 whole population is initialized at 0.5. 1000 loci are simulated in total and they are

185 separated in 200 chunks of 5 SNPs in physical linkage with the recombination rate

186 between adjacent loci fixed at 0.1 . Three different quantitative traits are coded by a

187 group of 10 different loci (quantitative trait locus, QTL). The first quantitative trait is

188 coded by the loci $1,11,21, \ldots, 91$. The trait value is simply the sum of the genotype

189 values and therefore can take values between 0 and 20 . We add to each trait a

190 random noise (nonheritable variation) drawn from a normal distribution $N(0,2)$. The

191 second quantitative trait is coded by loci $101,111, \ldots, 191$ and the third is coded by

192 loci 201, 211,..., 291. Each quantitative trait is therefore coded by 10 independent

193 SNPs (QTL), resulting in a total of 30 causal SNPs among the 1000. Selection can

194 have an effect on linked loci, for instance, loci 2, 3, 4 and 5 can be impacted by

195 selection on locus 1 . However, recombination is high enough (0.1) to expect a limited

196 linkage effect. We have defined 10 different environmental variables. The first one

197 determines the selective pressure on trait 1, the second one on trait 2 and the third

198 one on trait 3. The first environment variable is a quadratic gradient coded by

199 function env1 $\left.=-(\cos (\theta) *(\mathrm{i}-3.5))^{2}-(\sin \theta) *(\mathrm{j}-3.5)\right)^{2}+18, \theta=\pi / 2$, with $\mathrm{i}$ and $\mathrm{j}$ being

200 the population indicator on the $8 \times 8$ lattice. The second one is a linear plan gradient 
201 coded by function env2 $=\mathrm{h}^{*} \cos (\theta)^{*}(\mathrm{i}-1)+\mathrm{h}^{*} \sin (\theta)^{*}(\mathrm{j}-1)+k$ with $h=2, \theta=\pi / 4$ and

$202 k=3$. The third environment variable simulates a coarse environment with value 203 env3 = 2 for all populations except population $(\mathrm{i}, \mathrm{j})=(2,2),(2,3),(3,2),(3,3),(6,2)$, $204(6,3),(7,2),(7,3),(2,6),(2,7),(3,6),(3,7),(6,6),(6,7),(7,6),(7,7)$, for which env3 $=18$.

205 Env4, env5 and env6 have exactly the same equation as env1, env2 and env3, 206 respectively, except for a noise term explained below. The remaining 4 environment 207 variables are similar to env2 but with different values of $\mathrm{h}$ and $\theta$. Env7 has $h=2, \theta=$ 2080 and $k=3$. Env8 has $h=2, \theta=\pi / 4$ and $k=0$. Env9 has $h=1, \theta=\pi / 4$ and $k=4$. Env10 209 has $h=0.5, \theta=\pi / 4$ and $k=8$. Graphical representation of the mean environmental 210 value is given in Fig. 2. Environmental variables 4, 5 and 6 have, respectively, the 211 same mean value spatial distribution as environmental variables 1,2 and 3 . The 212 environmental equation gives a mean value of the environment variable. To avoid 213 collinearity between environmental variables, we added noise by drawing an 214 environment value within a normal distribution $\mathrm{N}(\mu=$ env, $\sigma=1)$. The fitness for 215 each trait is set to be $-\exp (\mathrm{x}-\mathrm{env})^{\wedge} 2 /\left(2^{*} \omega^{\wedge} 2\right)$, with $x$ being the quantitative trait 216 value, env the environmental value and $\omega$ is the defining selection strength and has 217 been set to 20 , which was found to be enough for loci to be often detected. To get the 218 overall fitness for a given individual, the fitness associated with each trait is 219 multiplied. Fitness values are used to determine the number of offspring during the 220 simulations, which have been launched for 500 generations. At the end of the 
221 simulation, we sampled 10 individuals per population resulting in 640 individuals

222 with 1000 SNP-like loci.

223

224 3. Real dataset

225

The Populus trichocarpa dataset is a sample of 424 individuals genotyped on

22633,070 SNPs from 25 drainages (i.e., topographic units separated by watershed

227 barriers) (Geraldes et al., 2014). Genotyping of each accession was performed with a

228 34K Populus SNP array targeting 34,131 SNPs mostly within (plus $2 \mathrm{~kb}$ upstream

229 and downstream) 3543 genes (Geraldes et al., 2013). A total of 21 climatic variables

230 are available on each sampling site (Table S1). From the 33,070 SNPs, we removed

231 the SNPs with missing values and a minor allele frequency below 5\% resulting in

23217,224 SNPs.

233 We explored the adaptive genetic variability of the Populus trichocarpa

234 dataset using PCA-based (pcadapt) and RDA-based method using the 21

235 environmental variables as explanatory variables (Table S2). Similar to the

236 simulations above, we created two environmental composite variables by keeping

237 the two first axes of a PCA on the 21 environmental variables. We then used the

238 latent factor mixed model (LFMM) procedure on these composite variables and with

239 one latent factor $(K=1)$. We finally compared the list of outliers found with these

240 three approaches to the ones found using Fst based methods (Geraldes et al., 2014). 
We then performed a second RDA with only the loci previously found as

242 outliers ( $q$-values $<0.1$ ). This set of outliers gives an "adaptively enriched genetic

243 space" (Steane et al., 2014) and using a second RDA on these specific loci, we can

244 identify the environmental variables that are the most correlated with "putatively"

245 adaptive variation. Using only outlier loci avoids potential interferences brought by

246 the "neutral" genetic variation during the alignment between genetic and

247 environmental variation performed by RDA. We then used the scores of the different

248 Populus trichocarpa populations along the first two RDA axes to build composite

249 indexes, which correspond to the main environmental pressures driving adaptive

250 genetic variation. The individuals' scores on the axes of the adaptively enriched

251 genetic RDA can be interpreted as cursors of individual genetic adaptation to the

252 environmental variables associated with these axes. We thus represented the mean

253 RDA1 and RDA2 individual score for each of the 133 populations on a map. It allows

254 visualizing the adaptive landscape across the sampling area.

255

256 RESULTS

257 1. Genome scan on simulated dataset using RDA, pcadapt and LFMM 258 methods

259 The procedure was first performed on one simulation (sim1) with pcadapt, 260 RDA and LFMM approaches. To perform RDA, we considered environmental 
261 variables 1-10 as explanatory variables. For pcadapt and RDA, we retained the first

262 four ordination axes to compute Mahalanobis distances as indicated by the scree

263 plots (Fig. S1). In parallel, we independently launched LFMM analysis with the first

264 two PCs resulting from a PCA applied to environmental variables (Fig. S2). We then

265 estimate that a locus is considered as an outlier by LFMM if there is a significant

266 correlation with at least one of the two composite variables.

267 The software package pcadapt is successful at detecting QTL2 SNPs (Fig. 3

268 topleft) but fails entirely at detecting QTL1 and QTL3 SNPs. The RDA-based

269 approach also detects QTL2 SNPS and most of the QTL1 SNPs in contrast to pcadapt

270 (Fig. 3 topright). The LFMM method is also able to detect most of the QTL1 and all

271 the QTL2 SNPs. These SNPs are detected when respectively regressing the genetic

272 dataset with the first and the second environmental composite variables (Fig. 3

273 bottom). For the SNPs associated with QTL3, the three methods have negligible

274 power to detect them.

275 When looking at the projection of each SNP in the RDA space, we can detect

276 links between QTL SNPs and environmental variables (Fig. 4). RDA1 is strongly

277 correlated to env2 and env5 and the QTL2 SNP show extreme scores on this axis

278 compared to the other SNPs, especially the neutral ones (Fig. 4A). RDA2 is also

279 associated with env7, env8, env9 and env10, which had not been used as direct

280 selective drivers during the simulations. RDA3 is correlated to env1 and env4 
281 corresponding to QTL1 SNPs and there is also a weak association between RDA4

282 (env3 and env6) and the SNPs associated with QTL3 (Fig. 4B \& 3C).

283 When looking at the percentage of environmental variance explained by the

284 RDA and PCA axes, we find that the first four RDA axes explain more variance of each

285 environmental variable than the first four axes of the PCA (Fig. 5). The difference is

286 especially true for env1 and env4, which are strongly associated with the first four

287 axes of the RDA, whereas PCA axes show an almost null correlation. The variables

288 env7, env8, env9 and env10 are also correlated to the first four axes of the RDA,

289 whereas PCs show far less correlation with these environments. Finally, RDA axes

290 show more correlation with the env3 and env6 variables than PCs $\left(\mathrm{R}^{2}=\sim 0.30\right.$ vs $\mathrm{R}^{2}$

$291=\sim 0$ ). Furthermore, each RDA axis, from the first one to the fourth one, is associated

292 with more than one environmental variable, whereas only one PC (PC1) is

293 associated with env2 and env5 (Fig. S3).

294

295

Finally, over the 100 simulations, we measured the average FDR and power

296 for pcadapt, LFMM and RDA (Fig. 6). The three methods correctly identify almost all

297 of the QTL2 SNPs (Fig. 6A). RDA-based analysis and LFMM are more powerful than

298 pcadapt to identify QTL1 SNPs with, respectively, 75\%, 48\% and less than 10\% of

299 true identification. For QTL3, pcadapt is not able to find any of the SNPs under

300 selection, whereas RDA reaches $24 \%$ of true identification and LFMM reaches $22 \%$. 
301 The three methods properly control for the false discovery rate because the

302 proportion of false discoveries obtained with pcadapt, LFMM and the RDA-based 303 methods are, respectively, 5\%, 2.3\% and 2.3\% when controlling for a nominal error 304 rate of $5 \%$.

\section{Populus trichocarpa}

For the RDA and pcadapt analysis on the P. trichocarpa dataset, we retained 308 respectively $K=3$ and $K=6$ axes as indicated by the scree plots (Fig. S4) and we used 309 the two first axes of a PCA on environmental variables as composite variables for the 310 LFMM procedure (Fig. S5). Analysis with an FDR of 0.1 provides a list of 151 outliers

311 for RDA, 117 for pcadapt and 36 for LFMM. Interestingly, the three methods have 312 only 9 outliers in common and 117, 77 and 11 SNPs are outliers, respectively, 313 specific to RDA, pcadapt and LFMM.

314 When we compared RDA genome scan results with the outliers found by 315 Geraldes et al. (2014) based on Fdist, BayeScan and bayenv methods, we found a 316 substantial overlap between them (Fig. 7). Among the 151 outliers found with the 317 RDA-based method, 69 have already been picked up by Fst-based methods (see 318 Table S3). Interestingly, the loci showing the lowest p-values with the RDA analysis 319 are also pointed out as outliers by the Fst and pcadapt methods, but not by the 320 LFMM procedure (Fig. 7). However, the RDA-based genome scan found 72 SNPs that 
321 no other methods, including pcadapt, detected as outliers.

323 the RDA-based genome scan method (adaptive enriched genetic space), we found 324 that the two first axes keep most (respectively $40 \%$ and $18 \%$ ) of the adaptive 325 genetic variance among the populations (Fig. 8A). These two axes correspond to the 326 two principal selective pressures in the sampling area. RDA1 is correlated with 327 several environmental variables used in the analysis (Fig. 8A), including DD_0, DD18, 328 MAT, MCMT, and EMT, which correspond to temperature indexes. RDA1 is also 329 correlated to moisture variables (e.g., AHM or MAP), continentality (TD) and 330 flowering period length (bFFP and eFFP). This axis summarizes a gradient of global 331 climatic variation encountered in the sampling area with southern and coastal 332 populations opposed to northern and continental populations (Fig. 8B). On the other 333 side, RDA2 also correlates with moisture and temperature variables such as AHM, 334 MAP, TD, MCMT, and eFFP and contrasts individuals from coastal locations to 335 individuals from inland areas (Fig. 8C).

339 detect true positives when environmental gradients related to adaption are 340 correlated to population structure. For instance, QTL2 SNPs are adaptive along an 
341 environmental gradient that is correlated with population structure (see Fig. 2) and

342 they are detected by pcadapt (Fig. 6). By contrast, when the environmental gradient

343 is not correlated to population structure (QTL3 and QTL1 to a lesser extent) it fails

344 to detect adaptive SNPS. In this case, PCA ordination is not capable of orienting

345 genetic variation into the direction of environment 1 or 3. Fig. 6 shows that RDA has

346 a larger statistical power than pcadapt to detect QTL1 SNPs by taking advantage of

347 information from environmental local conditions. It can be attributed to a better

348 alignment between the genetic space and the environmental variable improving the

349 power to detect true positives. This better alignment is easily visible when looking at

350 the PCA and RDA correlation with environmental variables (Fig. 5 \& Fig. S3). PCA is

351 only capable of identifying axes, which correspond to maximum genetic

352 differentiation (PC1 and PC2), whereas RDA is also able to capture the genetic

353 variability associated with other environmental variables, such as env1 (RDA3) and

354 to a lesser extent env3 (RDA4).

355

Genotype-environment approaches (GEA) explicitly take into account the

357 relationship between genotypes and the environment (Joost et al. 2007, de

358 Villemereuil et al. 2014, Lotterhos and Whitlock 2015). We showed that univariate

359 GEA, implemented in LFMM, and multivariate GEA, based on RDA, can show similar

360 results in identifying adaptive variation although RDA is more powerful than LFMM 
361 for QTL1 SNPs (Fig. 6). As suggested in the literature, we limited the number of

362 environmental predictors during the LFMM procedure by using environmental PCA

363 axes as composite variables (Frichot et al., 2013). We then only used the two first

364 PCs, but adding the third and fourth ones could potentially increase the capacity of

365 LFMM to detect QTL1 and QTL3 SNPs. It points out one of the advantages of a

366 multivariate procedure (e.g., RDA) compared to univariate GEA because multivariate

367 methods take all environmental variation into account at the same time and can

368 simultaneously detect associations between different sets of loci and different sets of

369 environmental variables (Forester et al., 2017).

Both RDA and LFMM fail to detect QTL3 outliers in, respectively, 75\% and

$37280 \%$ of the simulations (Fig. 6), possibly because of the additional difficulty in

373 detecting adaptive SNPs on a coarse environment compared to a smooth

374 environmental gradient as in environment 1 and 2. LFMM and the RDA-based

375 genome scan rely on linear association between the environment and allelic

376 abundance $(0,1$ or 2$)$. Patchy selective pressure, such as env3, is difficult to identify

377 with a correlative approach. Forester and colleagues (2015) already found that

378 heterogeneous environmental selection decreases the capacity of RDA to identify the

379 loci under selection. The strength of selection and the level of allelic dispersion

380 during the simulations strongly influence the statistical power of RDA; weak 
381 selective strength and strong dispersion are the less favorable conditions (Forester

382 et al., 2015). Nonetheless, our simulations plead in favor of using a constrained

383 ordination method when relevant environmental variables are available in order to

384 both orientate the ordination axis in the direction of environmental gradients and to

385 account for most of the environmental variation. This result confirms that

386 constrained ordination shows a superior combination of low false positive and high

387 true positives rates across all levels of selection (Forester et al., 2017).

388 During our RDA-based procedure, we use Mahalanobis distances as a test

389 statistic to identify genetic variation significantly associated with environmental

390 gradients. This distance is computed based on the scores of more than one RDA axis

391 and can thus condense the information of several RDA axes in one statistic. Some

392 other studies have used different RDA-based statistics to identify the loci under

393 putative selection. They often used a value estimated independently for the different

394 RDA axes, such as the loadings of the loci along the axis (e.g., extreme squared

395 loadings for Latsky et al., 2012) and considering a threshold such as \pm 3 SD (Forester

396 et al., 2017). Mahalanobis distances can capture complex selection patterns where

397 allelic variation depends on more than one environmental gradient. This statistic is

398 appropriate to identify selective pressures on natural populations, which usually

399 encompass multifactorial gradients of selection (Salmela, 2014). 
One application of ordination methods is to detect selective gradients. The

402 RDA-based approach succeeded very well in decomposing the QTL SNPS-

403 environments associations along the different axes, at least in our simulations. In our

404 example, we succeeded in dissociating the outliers linked to the different

405 environmental variables, and thus to precisely identify which set of variables drives

406 each part of the adaptive genetic variability (Fig. 4). RDA1 is strongly associated with

407 env2, RDA2 with env7 (collinear with isolation by distance pattern in the grid),

408 RDA3 with env1 and RDA4 with env3. As expected, the correlated environments are

409 also strongly associated with these respective axes. This is reflecting the fact that it

410 is difficult to distinguish among several collinear variables which environmental

411 variable has a causal effect on the individual fitness. However, it is often sufficient to

412 identify the combination of environment variables having a strong association with

413 adaptive variation without precisely knowing the underlying mechanical process.

414 These simulations serve as a proof of concept to show that ordination axes can

415 provide holistic measures of genomic adaptation (Steane et al., 2014). Under this

416 idea, scores of individuals on one RDA axis reflect their degree of adaptation along

417 this axis. RDA produces useful visualization of gradients of genetic adaptation (Fig. 418 8). 
421 (72) that are not detected by any other genome scan method, including pcadapt and

422 LFMM (Fig. 7 and Table S3). These results support the simulation analysis

423 conclusions that the RDA-based genome scan is able to pick up more genetic

424 markers in relation to environmental gradients than pcadapt. It is, however,

425 noticeable that a proportion of outliers are shared between PCA and RDA-based

426 methods (29), highlighting the fact that both approaches are partially similar. The

427 difficulty in interpreting the PCA axes is compensated by the fact that it is a blind

428 method in regard to environmental data, so it can pick up genes that we will miss

429 using RDA because some crucial environmental data are lacking. In the same vein,

430 half of the outlier loci found with the LFMM procedure (14 over 33 loci) are common

431 with RDA results (Fig. 7 and Table S3). The two methods rely on linear regression

432 between environmental and genetic variability, and by using PCs as composite

433 environmental variables in the LFMM procedure, we even add some similarity

434 between the two approaches.

435 After focusing the RDA analysis on the loci showing significant association

436 with environmental variables (151 outliers), we identified the predominant

437 environmental gradients structuring the genetic adaptation of Populus trichocarpa.

438 The main one is a composite climatic variation (RDA1 in Fig. 8), gathering

439 precipitation, temperature and seasonality variations. It clearly contrasts the

440 populations coming from the extreme northern part of the sampling area (Alaska) 
441 and the population coming from the inland locations (higher altitudes) to the

442 populations close to the coast in British Columbia (Canada) and the southern

443 populations, near Portland, Oregon (USA). This result is in accord with the

444 conclusions of Geraldes et al. (2014). In their study, they point out the need of

445 adaptation to seasonality, photoperiod and frost events for Populus trichocarpa

446 along its continuous distribution from 44 to almost 60 degrees of latitude. RDA2 is

447 associated with moisture regimes and points out a gradient of adaptation between

448 coastal locations, strongly watered, and inland populations, receiving less

449 precipitation (Fig. 8). These two environmental gradients are the strongest selective

450 pressures driving Populus trichocarpa climatic adaptation in northwest America,

451 considering the set of environmental variables available and the scale of the analysis.

452 To go further, these composite indexes could also serve to predict (i) provenances

453 that would perform well in common gardens; (ii) patterns of adaptation to local

454 climate across the geographic range; or (iii) future adaptive landscapes in the

455 context of climate change.

456

\section{ACKNOWLEDGEMENTS}

458 We would like to dedicate this paper to the memory of our colleague and

459 beloved friend Eric Bazin (1977-2017) who passed away too soon. 
461 0004) and MB acknowledges the Grenoble Alpes Data Institute, which is supported

462 by the French National Research Agency under the "Investissements d'avenir"

463 program (ANR-15-IDEX-02). MB also acknowledges support from the ANR project

464 AGRHUM (ANR-14-CE02-0003-01).

466 REFERENCES

467 Bazin, Eric, Kevin J. Dawson, and Mark A. Beaumont. 2010. "Likelihood-Free Inference of Population Structure and Local Adaptation in a Bayesian Hierarchical Model." Genetics 185 (2): 587-602.

Coop, Graham, David Witonsky, Anna Di Rienzo, and Jonathan K. Pritchard. 2010. "Using Environmental Correlations to Identify Loci Underlying Local Adaptation.” Genetics 185 (4): 1411-23.

Kort, Hanne De, Katrien Vandepitte, Hans Henrik Bruun, Déborah Closset-Kopp, Olivier Honnay, and Joachim Mergeay. 2014. "Landscape Genomics and a Common Garden Trial

475 Reveal Adaptive Differentiation to Temperature across Europe in the Tree Species Alnus Glutinosa." Molecular Ecology 23 (19): 4709-21.

De Villemereuil, Pierre and Oscar E. Gaggiotti. 2015. “A New FST-Based Method to Uncover Local Adaptation Using Environmental Variables." Methods in Ecology and Evolution 6 (11): 1248-58. doi:10.1111/2041-210X.12418.

Duforet-Frebourg, Nicolas, Keurcien Luu, Guillaume Laval, Eric Bazin, and Michael G B Blum. 2016. "Detecting Genomic Signatures of Natural Selection with Principal Component Analysis: Application to the 1000 Genomes Data." Molecular Biology and Evolution 33 (4): 1082-93.

Foll, Matthieu, and Oscar Gaggiotti. 2008. "A Genome-Scan Method to Identify Selected Loci Appropriate for Both Dominant and Codominant Markers: A Bayesian Perspective." Genetics 180 (2): 977-93.

487 Forester, Brenna R, Jesse R Lasky, Helene H Wagner, and Dean L Urban. 2017. "Comparing 488 Methods for Detecting Multilocus Adaptation with Multivariate Genotype,” no. 970: 1-42. 
Forester, Brenna R., Matthew R. Jones, Stéphane Joost, Erin L. Landguth, and Jesse R. Lasky. 2016. "Detecting Spatial Genetic Signatures of Local Adaptation in Heterogeneous Landscapes." Molecular Ecology 25 (1): 104-20.

François, Olivier, Helena Martins, Kevin Caye and Sean D. Schoville. 2016. "Controlling false discoveries in genome scans for selection." Molecular Ecology 25 (2): 454-469.

Frichot, Eric, Sean D. Schoville, Guillaume Bouchard, and Olivier François. 2013. "Testing for Associations between Loci and Environmental Gradients Using Latent Factor Mixed Models." Molecular Biology and Evolution 30 (7): 1687-99.

Geraldes, A., S. P. Difazio, G. T. Slavov, P. Ranjan, W. Muchero, J. Hannemann, L. E. Gunter, et al. 2013. "A 34K SNP Genotyping Array for Populus Trichocarpa: Design, Application to the Study of Natural Populations and Transferability to Other Populus Species." Molecular Ecology Resources 13 (2): 306-23.

Geraldes, Armando, Nima Farzaneh, Christopher J. Grassa, Athena D. Mckown, Robert D. Guy,

502

503

504 Shawn D. Mansfield, Carl J. Douglas, and Quentin C B Cronk. 2014. "Landscape Genomics of Populus Trichocarpa: The Role of Hybridization, Limited Gene Flow, and Natural Selection in Shaping Patterns of Population Structure." Evolution 68 (11): 3260-80.

Hecht, Benjamin C., Andrew P. Matala, Jon E. Hess, and Shawn R. Narum. 2015. "Environmental Adaptation in Chinook Salmon (Oncorhynchus Tshawytscha) throughout Their North American Range." Molecular Ecology 24 (22): 5573-95.

Hoban, Sean, Joanna L. Kelley, Katie E. Lotterhos, Michael F. Antolin, Gideon Bradburd, David B. Lowry, Mary L. Poss, Laura K. Reed, Andrew Storfer, and Michael C. Whitlock. 2016. "Finding the Genomic Basis of Local Adaptation: Pitfalls, Practical Solutions, and Future Directions." The American Naturalist 188 (4): 379-97.

517 Luu, Keurcien, Eric Bazin, and Michael G B Blum. 2017. "Pcadapt: An R Package to Perform

Lasky, Jesse R., David L. Des Marais, John K. McKay, James H. Richards, Thomas E. Juenger, and Timothy H. Keitt. 2012. "Characterizing Genomic Variation of Arabidopsis Thaliana: The Roles of Geography and Climate." Molecular Ecology 21 (22): 5512-29.

Legendre, Pierre, and Louis Legendre. 1988. "Numerical Ecology, Volume 24." (Developments in Environmental Modelling) 24: 870.

520 Martins, Helena, Kevin Caye, Keurcien Luu, Michael G.B. Blum, and Olivier François. 2016.

521 "Identifying Outlier Loci in Admixed and in Continuous Populations Using Ancestral 522 Population Differentiation Statistics.” Molecular Ecology 25 (20): 5029-42. 
523

524

525

526

527

528

529

530

531

532

533

534

535

536

537

538

539

540

541

542

543

544

545

546

547

548

549

550

551

552

553

554

Oksanen, Jari, F Guillaume Blanchet, Michael Friendly, Roeland Kindt, Pierre Legendre, Dan Mcglinn, Peter R Minchin, et al. 2015. "Vegan: Community Ecology Package." R Package Version 2.3-2, https://CRAN.R-project.org/package=vegan.

Peng, Bo, and Marek Kimmel. 2005. "simuPOP: A Forward-Time Population Genetics Simulation Environment." Bioinformatics 21 (18): 3686-87.

Pritchard, Jonathan K., and Anna Di Rienzo. 2010. "Adaptation - Not by Sweeps Alone." Nature Reviews Genetics 11 (10). Nature Publishing Group: 665-67.

Salmela, Matti J. 2014. "Rethinking Local Adaptation: Mind the Environment!" Forest Ecology and Management 312. Elsevier B.V.:271-81.

Savolainen, Outi, Martin Lascoux, and Juha Merilä. 2013. "Ecological Genomics of Local Adaptation." Nature Reviews Genetics 14 (11): 807-20.

Steane, Dorothy A., Brad M. Potts, Elizabeth McLean, Suzanne M. Prober, William D. Stock, René E. Vaillancourt, and Margaret Byrne. 2014. "Genome-Wide Scans Detect Adaptation to Aridity in a Widespread Forest Tree Species." Molecular Ecology 23 (10): 2500-2513.

Storey, John D. and Andrew J. Bass. 2011. "Bioconductor's Qvalue Package.” Gene, no. 2001: $1-6$.

Vangestel, Carl, Andrew J. Eckert, Jill L. Wegrzyn, J. Bradley St. Clair, and David B. Neale. 2018. "Linking Phenotype, Genotype and Environment to Unravel Genetic Components Underlying Cold Hardiness in Coastal Douglas-Fir (Pseudotsuga Menziesii Var. Menziesii)." Tree Genetics \& Genomes 14 (1). Tree Genetics \& Genomes:10.

Vatsiou, Alexandra I., Eric Bazin, and Oscar E. Gaggiotti. 2016. "Detection of Selective Sweeps in Structured Populations: A Comparison of Recent Methods." Molecular Ecology 25 (1): 89-103.

Wang, Jiahui, Ruben Zamar, Alfio Marazzi, Victor Yohai, Matias Salibian-Barrera, Ricardo Maronna, Eric Zivot, et al. 2014. "Robust: Robust Library." $R$ Package Version 0.4-16. http://cran.r-project.org/package=robust.

Whitlock, Michael C., and Katie E. Lotterhos. 2015. "Reliable Detection of Loci Responsible for Local Adaptation: Inference of a Null Model through Trimming the Distribution of $F$ ST." The American Naturalist 186 (S1): S24-36.

Yang, Wen Yun, John Novembre, Eleazar Eskin, and Eran Halperin. 2012. "A Model-Based Approach for Analysis of Spatial Structure in Genetic Data." Nature Genetics 44 (6): 725 31.

555 


\section{DATA ACCESSIBILITY STATEMENT}

557

558

559

560

561

562

563

564

565

566

\section{7}

568

569

570

571

572

573

574 under selection.

575 Figure 2: Graphical representations of mean environmental values for 576 environments 1 to 10 . Equation determining the environment is given in the main 577 manuscript. Mean environmental values for environments 4, 5 and 6 are, 578 respectively, equivalent to environments 1,2 and 3.

579 Figure 3: Manhattan plots of the results obtained with pcadapt (upper left panel), 580 RDA (upper right panel), and LFMM (lower panels) for one simulated dataset. 
581 Figure 4: Projection of SNPS and environmental variables into the RDA space.

582 Figure 5: Proportion of environmental variance explained by the first four 583 ordination axes using RDA or PCA. The values correspond to the r-squared of the 584 regression between the environmental variable and the first four axes of the 585 multivariate analysis.

586 Figure 6: Statistical power obtained with RDA, pcadapt, and LFMM. Estimate of 587 power corresponds to an average over 100 simulated datasets (error bars are 588 displayed). Power (A) is given separately for loci coding for quantitative traits 1, 2 589 and 3. False Discovery Rate (FDR) is given for all methods (B).

590 Figure 7: Scatter plot of the p-values returned by RDA and pcadapt procedures (A) 591 and the p-values returned by RDA and LFMM procedure (B) for the Populus 592 trichocarpa dataset. Black dots correspond to loci identified as outliers by Fst 593 methods in Geraldes et al. (2014). The dashed lines indicate a 10-3 p-value with RDA 594 and pcadapt or LFMM.

595 Figure 8: Projection of SNPs and environmental variables in the RDA performed on 596 the adaptively enriched genetic space (151 loci) of P. trichocarpa dataset (A). The 597 first two axes of the RDA projection represent 40\% (RDA1) and 18\% (RDA2) of the 598 explained variance. (B) and (C) show respective spatial representations of RDA1 and 599 RDA2 axes scores in the sampling area. The points represent the 133 sampled 600 populations and their color depends on the mean of individual RDA1 (B) and RDA2 601 (C) scores in the populations.

602 
604 Script S1: Detailed script of the RDA-based genome scan analysis.

605 Table S1: Environmental variables used in the Populus trichocarpa analysis.

606 Table S2: Climatic variable values for the 133 Populus trichocarpa sampled 607 populations.

608 Table S3: Comparison between RDA, pcadapt, fdist and bayescan outlier loci.

609 Figure S1: Scree plot for PCA and RDA analysis of the simulation 1 dataset.

610 Figure S2: PCA and scree plot of PCA on environmental variables of the first 611 simulation.

612 Figure S3: Heatmap of the $\mathrm{R}^{2}$ resulting from linear regression between the four first 613 axes of PCA and RDA analysis and the environmental variables.

614 Figure S4: Scree plot for PCA and RDA analysis of Populus trichocarpa dataset.

615 Figure S5: PCA and scree plot of PCA on environmental variables of the Populus 616 trichocarpa dataset. 


\section{Genotypes}

\begin{tabular}{|c|}
\hline $\begin{array}{lllll}0 & 0 & 1 & 2 & \ldots \\
0 & 0 & 1 & 1 & \ldots \\
0 & 1 & 2 & 2 & \ldots \\
1 & 0 & 1 & 1 & \ldots \\
0 & 0 & 1 & 2 & \ldots\end{array}$ \\
\hline
\end{tabular}

Center and scale

\section{Linear regression}

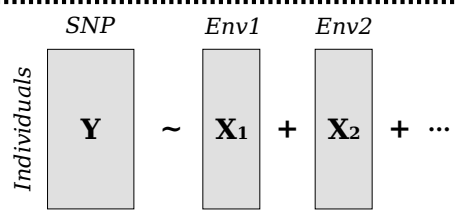

\section{Principal component analysis}

\section{Projection in the RDA space}
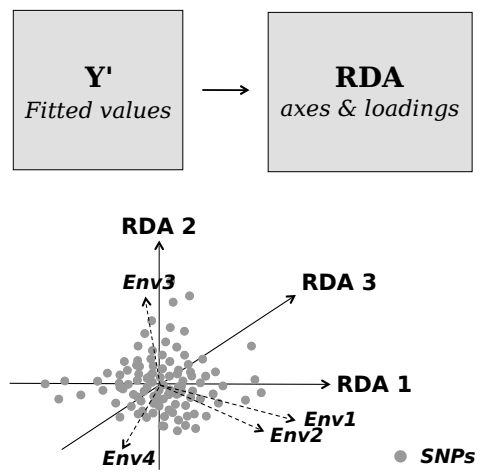

Recovering loci loadings on $K$ number of axes

\section{Mahalanobis distance (D)}

Correction by the genomic inflation factor

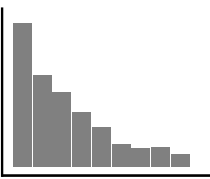

\section{P-values}

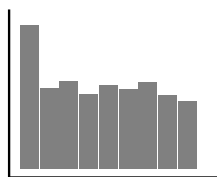

Adjusting for the false discovery rate (FDR)

\section{Outlier loci}

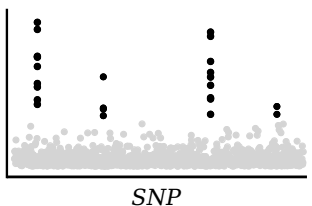


envir1

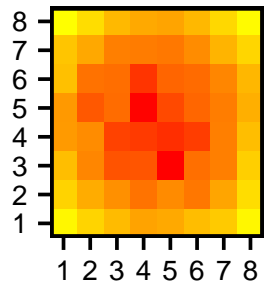

envir4

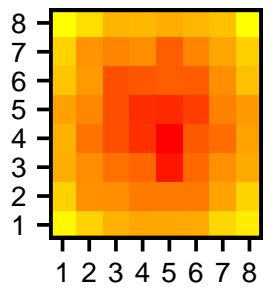

envir7

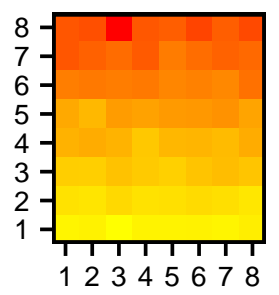

envir10

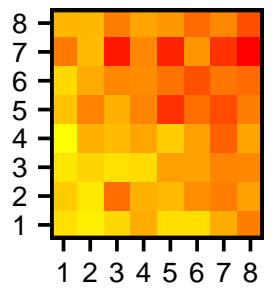

envir2

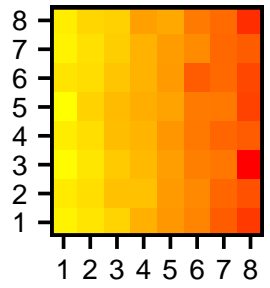

envir5

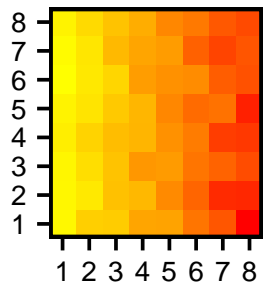

envir8

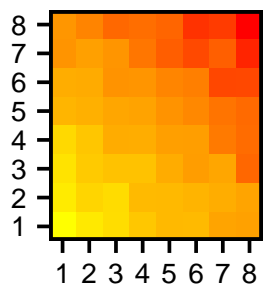

envir3

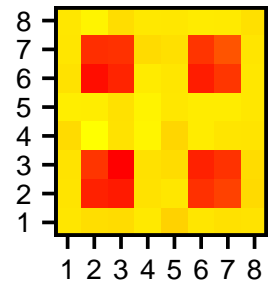

envir6

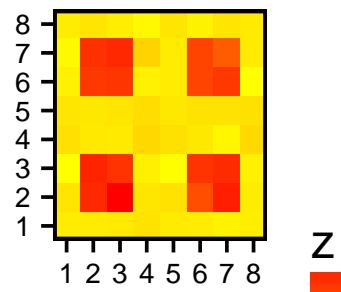

0
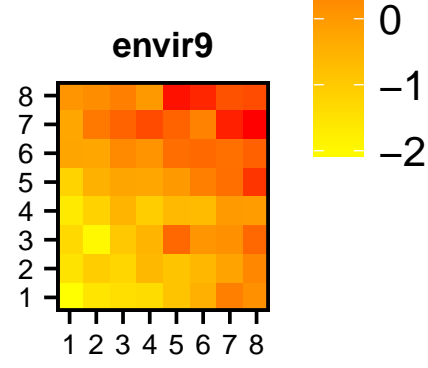
Selected Loci • QTL1 - QTL2 - QTL3
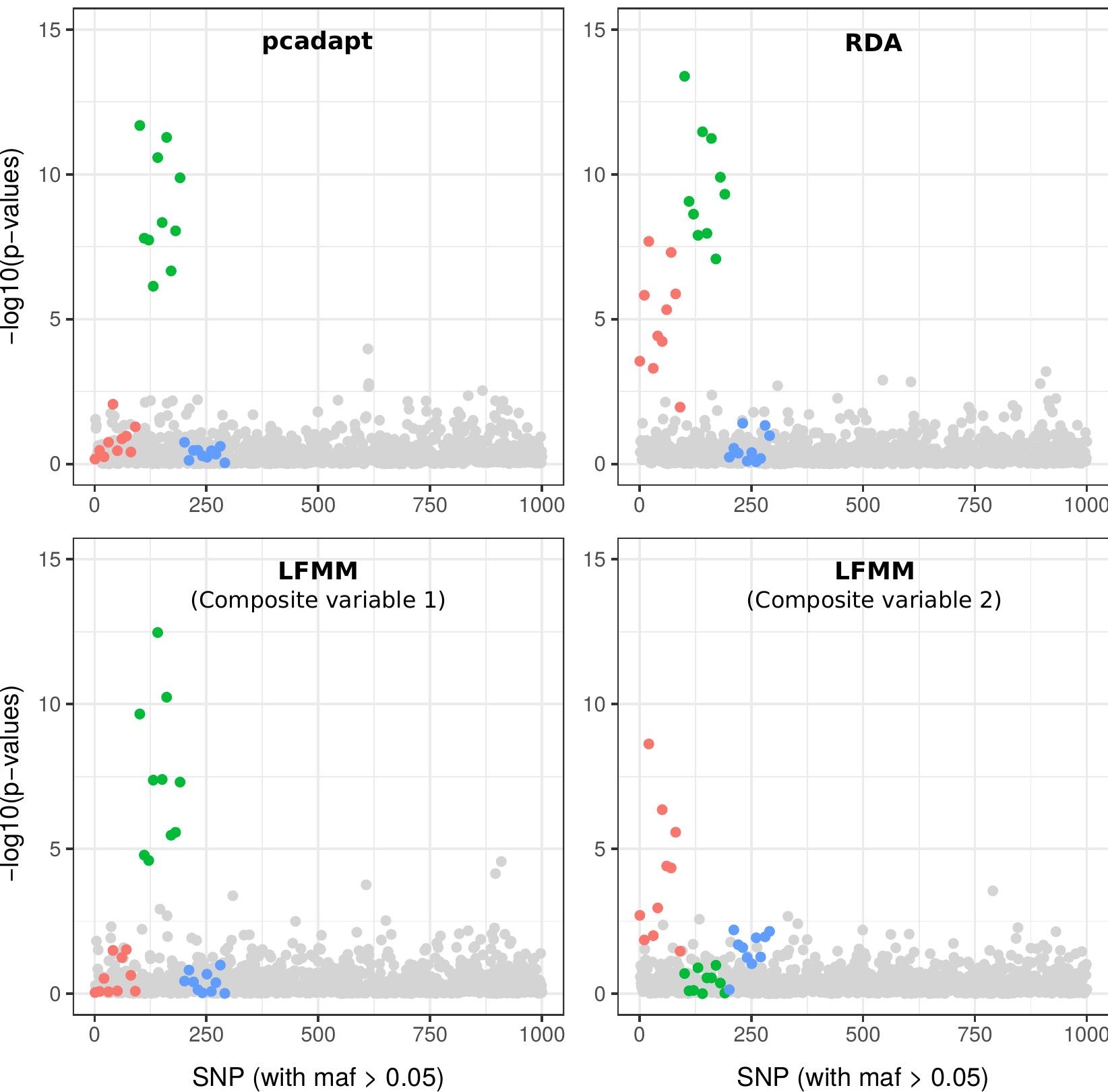


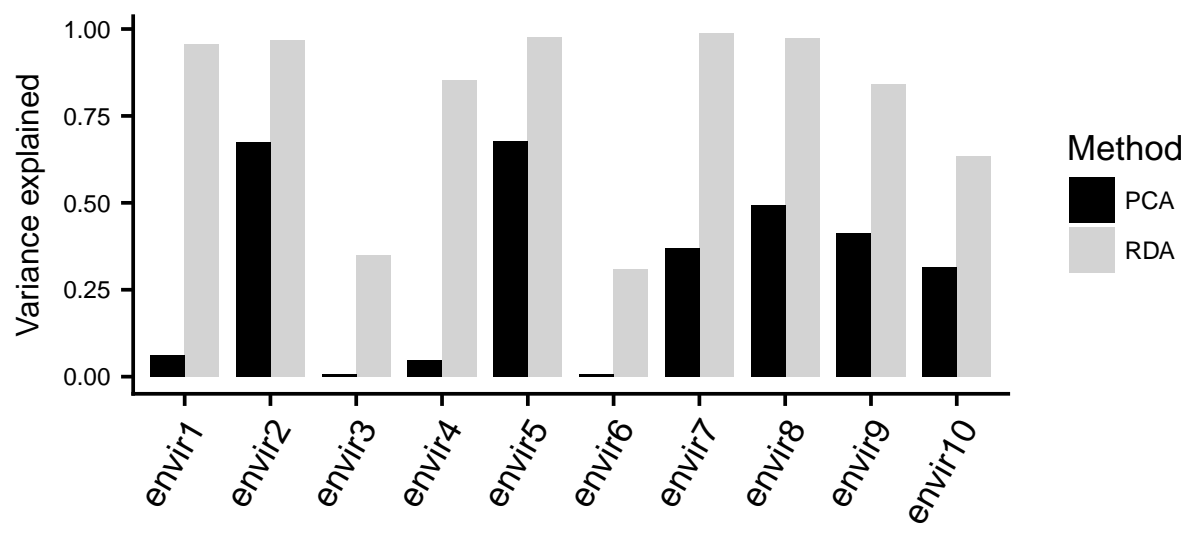




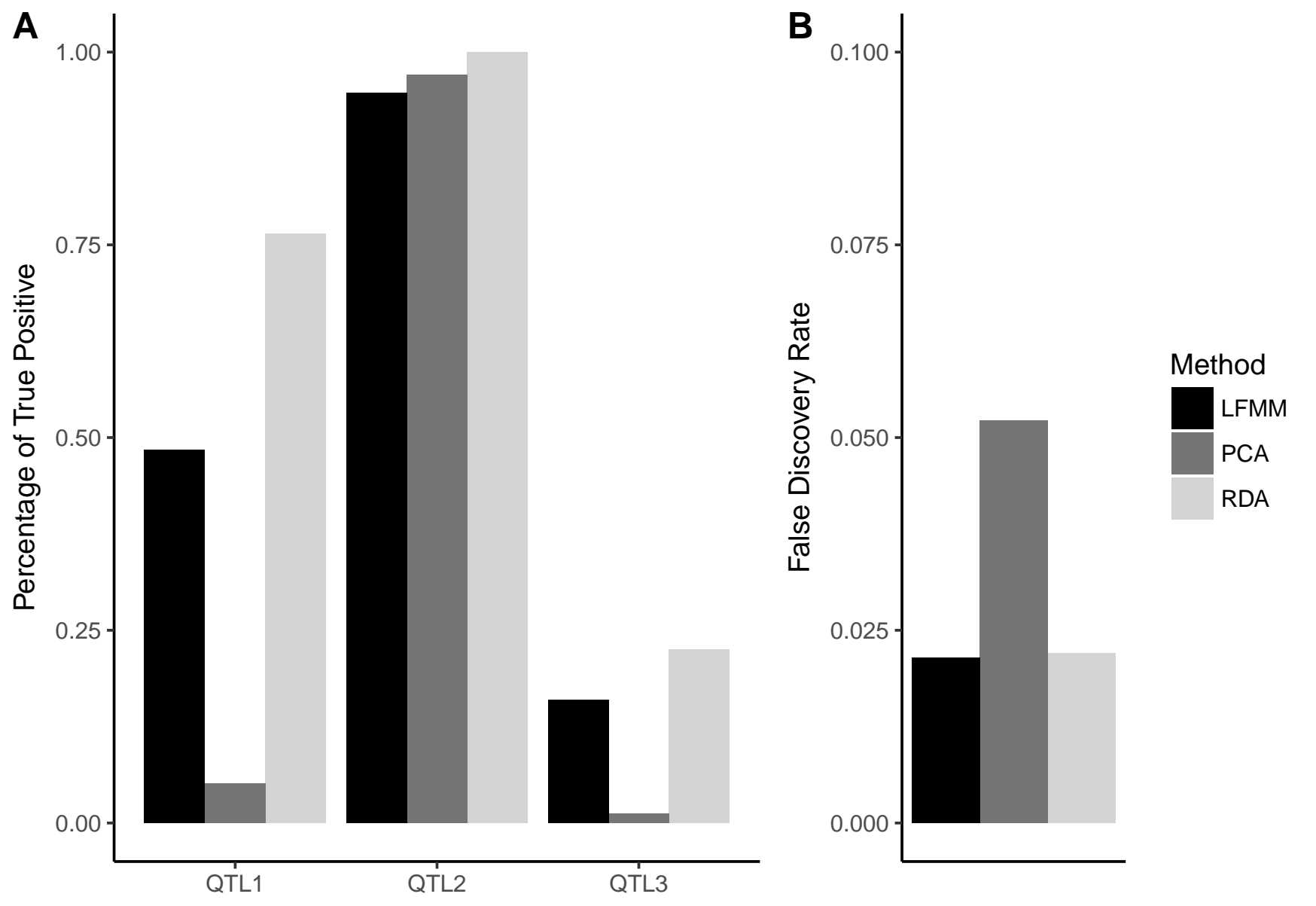


